\title{
Disparities in Adult African American Women's Knowledge of Heart Attack and Stroke Symptomatology: An Analysis of 2003-2005 Behavioral Risk Factor Surveillance Survey Data
}

\author{
MAY NAWAL LUTFIYYA, Ph.D., ${ }^{1}$ MARITES T. CUMBA, B.S., ${ }^{1}$ \\ JOEL EMERY MCCULLOUGH, M.D., M.P.H., M.S., ${ }^{2}$ ERIKA LAVERNE BARLOW, M.D., ${ }^{3}$ \\ and MARTIN S. LIPSKY, M.D., M.S. ${ }^{1}$
}

\begin{abstract}
Background: Heart disease and stroke are the first and third leading causes of death of American women, respectively. African American women experience a disproportionate burden of these diseases compared with Caucasian women and are also more likely to delay seeking treatment for acute symptoms. As knowledge is a first step in seeking care, this study examined the knowledge of heart attack and stroke symptoms among African American women.

Methods: This was a cross-sectional study analyzing 2003-2005 Behavioral Risk Factor Surveillance Survey (BRFSS) data. A composite heart attack and stroke knowledge score was computed for each respondent from the 13 heart attack and stroke symptom knowledge questions. Multivariate logistic regression was performed using low scores on the heart attack and stroke knowledge questions as the dependent variable.

Results: Twenty percent of the respondents were low scorers, and $23.8 \%$ were high scorers. Logistic regression analysis showed that adult African American women who earned low scores on the composite heart attack and stroke knowledge questions (range $0-8$ points) were more likely to be aged $18-34(\mathrm{OR}=1.36, \mathrm{CI} 1.35,1.37)$, be uninsured $(\mathrm{OR}=1.32, \mathrm{CI} 1.31,1.33)$, have an annual household income $<\$ 35,000(\mathrm{OR}=1.46, \mathrm{CI} 1.45,1.47)$, and have a primary healthcare provider $(\mathrm{OR}=1.22, \mathrm{CI} 1.20,1.23)$.

Conclusions: The findings indicated that knowledge of heart attack and stroke symptoms varied significantly among African American women, depending on socioeconomic variables. Targeting interventions to African American women, particularly those in lower socioeconomic groups, may increase knowledge of heart attack and stroke symptoms, subsequently improving preventive action taken in response to these conditions.
\end{abstract}

\footnotetext{
${ }^{1}$ Department of Family and Community Medicine, University of Illinois-Chicago College of Medicine at Rockford, Rockford, Illinois.

${ }^{2}$ Division of Environmental Health, Chicago Department of Public Health, Chicago, Illinois.

${ }^{3}$ University of Michigan Hospitals and Health Centers, OB/GYN Residency Program, St. Josephs Mercy Hospital, Ann Arbor, Michigan.
} 


\section{INTRODUCTION}

D ESPITE IMPROVEMENTS IN CARE, heart disease and stroke remain the first and third leading causes of death, respectively, for African American women. ${ }^{1,2}$ Stroke is also a major cause of adult disability for women in the United States. ${ }^{3,4}$ Nevertheless, a 2006 study by Christian et $\mathrm{al}^{5}$ found that awareness of heart disease/ heart attack as a leading cause of death was lower among African American women than Caucasian women ( $31 \%$ vs. $68 \%$ ). Moreover, findings from this same study indicated that women of all races/ ethnicities did not identify stroke as a leading cause of death for women in the United States. ${ }^{5}$

Timely care can reduce both the morbidity and mortality of heart attack and stroke. Delays in acute treatments, such as thrombolytic therapy and angioplasty, worsen the risk of death from heart attack. ${ }^{6-9}$ Similarly, there is a narrow window of opportunity for acute intervention in stroke patients. For optimum benefit, intravenous recombinant tissue plasminogen activator (rtPA) should be administered within 3 hours from the onset of ischemic stroke symptoms. ${ }^{9-12}$ Despite the benefits of early treatment for heart attack and stroke, many people still wait hours before seeking treatment, increasing their risk for long-term damage to the heart and brain. ${ }^{13}$

Gender and race are two variables known to affect risk and outcomes for acute vascular events, with African Americans overall experiencing the highest mortality rate from heart disease and an increased risk of stroke. ${ }^{1,2}$ In 2004, age-adjusted death rates per 100,000 for both heart disease and stroke were almost $40 \%$ higher for African American women than for white women: heart disease 236.5 vs. 172.9 and stroke 65.5 vs. $47.2 .^{2}$

Both African Americans and women have also been shown to routinely delay seeking treatment after the acute onset of symptoms of heart attack and stroke. ${ }^{14-17}$ Although, in general, most women can correctly identify symptoms of heart attack, there are ethnic differences in women's recognition of these symptoms, with non-Caucasians less likely than Caucasians to correctly identify symptoms. ${ }^{18}$ Similarly, although stroke awareness is increasing among women, a substantial gap of knowledge persists for African American women.,19 Despite evidence of gaps in knowledge and a disproportionate burden of disease, few studies have examined heart attack and stroke symptom awareness among African American women exclusively. Often, those studies including African American women treat them as a homogeneous group, either because of a small sample or the failure to consider the heterogeneity among a racial or ethnic group. By doing this, important within-group distinctions may be missed.

This study evaluated the current knowledge of heart attack and stroke symptoms among African American women and examined whether this knowledge varied by demographic factors and access to healthcare. This study stratified knowledge by the following variables: age, education, household income, health insurance status, having a primary care provider, and deferring medical care because of cost.

\section{MATERIALS AND METHODS}

Univariate, bivariate, and multivariate techniques were used to analyze a multiyear Behavioral Risk Factor Surveillance Survey (BRFSS) heart attack and stroke module database. BRFSS data are collected using a random-digit dial telephone survey targeting adults 18-99 years of age. These data are collected under the guidance of the Centers for Disease Control and Prevention (CDC) in collaboration with all U.S. states and most U.S. territories. Once collected, BRFSS data are weighted such that they are representative of the noninstitutional U.S. population by surveyed state. The data are cross-sectional and are focused on health risk factors and behaviors. A detailed description of the survey design and sampling measures can be found elsewhere. ${ }^{20}$

Data from the BRFSS optional module on heart attack and stroke were used in these analyses. Because different states use this module in different years, we merged 2003, 2004, and 2005 data to include as many states and territories as possible. In 2003, 17 states and United States Virgin Islands (USVI) included a module in their BRFSS surveys regarding symptoms of heart attack and stroke. In 2004, 7 states included the module; in 2005, it was included by 14 states and the District of Columbia. Data from 23 states, the USVI, and the District of Columbia were used in these analyses. If a state used the module more than once, only the data from the most recent year were used.

For the years in question, the BRFSS heart and stroke module included 13 questions focused on ascertaining knowledge on early symptoms of 
heart attack and stroke. Of these 13 questions, 6 were on knowledge of stroke symptoms, 6 were on knowledge of heart attack symptoms, and 1 question was on proper first response to either stroke or heart attack.

Respondents were asked if the following were warning signs of stroke: sudden confusion; trouble speaking or understanding; sudden numbness or weakness of face, arm, or leg; sudden trouble seeing in one or both eyes; sudden trouble walking, dizziness, or loss of balance or coordination; or sudden, severe headache with no known cause. An incorrect sign (i.e., sudden chest pain) was included to examine the possibility that respondents would answer "yes" for all the symptoms. Likewise, respondents were asked if the following were warning signs of a heart attack: pain or discomfort in the jaw, neck, or back; feeling weak, lightheaded, or faint; chest pain or discomfort; pain or discomfort in the arms or shoulders; shortness of breath. Just as was the case with stroke symptoms, an incorrect sign (i.e., trouble seeing in one or both eyes) was included to examine the possibility that respondents would answer "yes" for all the symptoms.

We chose to group the questions for heart attack and stroke symptomatology together for analysis because these disorders are both vascular events that share in common the need for prompt recognition of symptoms and prehospital action by either the patient or bystanders. Any costly public health campaign will likely need to address both these vascular diseases together, and strokes are often referred to as "brain attacks," as many aspects of early stroke management mimic heart attack management. However, to confirm that the assumption that knowledge among African American women for these vascular disorders was similar, we also examined heart attack and stroke questions separately and compared the scores for each to validate that knowledge scores for both diseases paralleled one another.

For analysis we computed a Heart Attack and Stroke Knowledge Score for each respondent. Correct answers received 1 point and were categorized according to the following scale: low score $2-8$ points, midrange score $9-10$ points, and high score 11-13 points. Although this scale, like most, is arbitrary, it served the purpose of allowing for the standardized comparison of knowledge levels among groups. We used a three-level scale in order to identify a low scor- ing population for comparison to a high scoring one. Correspondingly, for the descriptive analysis on the heart attack and stroke knowledge questions conducted separately from one another, a knowledge score was computed for each respondent for each distinct set of knowledge questions. As with the composite knowledge scores, for the individual knowledge components (heart attack and stroke separately), correct answers received 1 point. These scores were then categorized as either low or high scores according to the following scale: low scores $0-3$ points $(\leq 50 \%)$ and high scores $4-6$ points $(>50 \%)$. Unlike the composite knowledge scores that were split into three levels for analysis, the knowledge scores for the individual stroke and heart attack components were dichotomized because of fewer questions, resulting in a smaller range of scores.

Three original BRFSS variables-age, education, and annual household income-were recoded for this analysis. Age was recoded from a continuous variable to a categorical one with three factors/levels (18-34 years, 35-54 years, and $\geq 55$ years). For education and household income, recoding entailed the collapsing of multiple response categories into fewer ones-two factors for each variable. The two factors for education were $<$ high school and $\geq$ high school. Annual household income was categorized into the categories of $<\$ 35,000$ and $\geq \$ 35,000$. Three healthcare access variables: having a primary care provider, whether medical care was deferred because of cost, and health insurance status were also included in the analysis. The response categories of "don't know" and "refused to answer" were recoded as missing data.

A regression model was performed using low scores on the combined heart attack and stroke knowledge questions as the dependent variable. All adult African American women $\geq 18$ years were the population considered. The independent variables entered into the model were age, education, annual household income, health insurance status in the past 12 months, and deferring medical care in the past 12 months because of cost. Alpha was set at 0.05 for all tests of statistical significance. To further reduce bias, a constant was entered into the model. SPSS version 15.0 (SPSS, Chicago, IL) was used to complete the analyses to account for the complex survey design. The research was approved by the Internal Review Board of the University of IllinoisChicago College of Medicine at Rockford. 


\section{RESULTS}

The unweighted sample size for the population of interest for the years 2003-2005 was 11,269. For analysis, these data were weighted to represent 6,102,996 African American women aged $\geq 18$ years who answered questions on heart attack and stroke symptoms. Table 1 summarizes the characteristics of the study group. These data indicated that $<25 \%$ of the population was aged $\geq 55$ years, $83.7 \%$ had at least a high school education, and $67.1 \%$ lived in households with annual incomes $<\$ 35,000$. In almost equal proportions, $80.9 \%$ and $83.0 \%$ respectively, the population of interest had health insurance as well as an identified primary care provider. Almost $22 \%$ reported having deferred medical care because of cost some time in the past 12 months.

Table 2 presents the percentage of incorrect answers to the heart attack and stroke symptomatology questions for all adult African American female respondents. The number of incorrect answers for the questions ranged from a low of $6 \%$ for "Do you think chest pain or discomfort is a symptom of a heart attack?" to a high of $62.9 \%$ for "Do you think chest pain or discomfort is a symptom of a stroke?" For questions on heart attack symptoms, $48.3 \%$ of the respondents did not recognize that pain or discomfort in the jaw, neck, or back was a symptom of heart attack. In addition, $32.6 \%$ responded incorrectly to the question about feeling weak, lightheaded, or faint as a symptom of heart attack. They also incorrectly attributed sudden trouble seeing in one or both eyes as a symptom of heart attack $49.2 \%$ of the time. For questions regarding stroke symptomatology, respondents incorrectly identified sudden chest pain or discomfort as a symptom of stroke $62.9 \%$ of the time. A high proportion of respondents $(41.7 \%)$ also incorrectly identified severe headache with no known cause as not being a symptom of stroke. Finally, $13.2 \%$ failed to recognize that calling 911 was the appropriate first response to these acute events.

Table 3 describes the composite heart attack and stroke knowledge of symptomatology scores and also the knowledge scores for heart attack and stroke alone. Cumulative scores on the heart attack and stroke knowledge questions ranged from 2 to 13 points, with a mean score of 9.46 and a standard deviation (SD) of 1.73. Overall, the majority of adult African American women scored in the moderate range for the composite heart attack and stroke knowledge scores (56.3\%).
Table 1. Selected Characteristics of African American Women $\geq 18$ Years of Age Responding to the Heart and Stroke Module ${ }^{a}$ : 2003-2005 Behavioral Risk Factor Surveillance Survey Data ${ }^{\mathrm{b}}$

\begin{tabular}{lc}
\hline Variables and factors & $\%$ \\
\hline Age, years & \\
18-34 & 36.9 \\
$35-54$ & 38.8 \\
$\quad \geq 55-64$ & 24.3 \\
Education & \\
$\quad<$ High school & 16.3 \\
$\quad \geq$ High school & 83.7 \\
Health insurance & \\
$\quad$ Yes & 80.9 \\
$\quad$ No & 19.1 \\
Household income & 67.1 \\
$\quad<\$ 35,000$ & 32.9 \\
$\quad \geq \$ 35,000$ & \\
Medical care deferred because of cost & 21.7 \\
$\quad$ Yes & 78.3 \\
$\quad$ No & \\
Primary care provider & 83.0 \\
$\quad$ Yes & 17.0 \\
$\quad$ No & \\
\hline
\end{tabular}

a25 states/territories were included in this analysis. By year of data collection these were:

2003: Arkansas, Georgia, Nebraska, North Carolina, North Dakota, South Carolina

2004: Colorado, Connecticut, Kentucky, Ohio

2005: Alabama, DC, Florida, Iowa, Louisiana, Maine, Minnesota, Mississippi, Missouri, Montana, Oklahoma, Tennessee, US Virgin Islands, Virginia, West Virginia

${ }^{\mathrm{b}}$ Weighted $n=6,102,996$; unweighted $n=11,269$.

Twenty percent of the respondents were low scorers, and $23.8 \%$ were high scorers. When examining the heart attack alone and stroke alone knowledge scores, a higher proportion of African American adult women earned low scores on the composite heart attack knowledge questions in comparison to the composite stroke knowledge questions (17.8\% vs. $13.2 \%)$.

Bivariate analyses of the independent variables stratified by high/low composite heart attack and stroke knowledge scores were conducted (Table 4). All the relationships between the independent and dependent variables by score range proved to be statistically significant and were subsequently entered into a logistic regression model that used low scores on the heart attack and stroke knowledge questions as the dependent variable.

The multivariate logistic regression results are presented in Table 5. This analysis yielded that African American women aged $\geq 18$ years who earned low scores on the composite heart attack 
Table 2. Heart Attack and Stroke Knowledge Questions with Correct Responses by African American Women: 2003-2005 Behavioral Risk Factor Surveillance Survey Data ${ }^{\text {a }}$

Prologue Now I would like to ask you about your knowledge of the signs and symptoms of a heart attack and stroke. Which of the following do you think is a symptom of a heart attack (or stroke)?

For each, tell me "Yes," "No," or "Not sure"

Heart attack symptoms (correct answer)

Do you think pain or discomfort in the jaw, neck, or back is a symptom of a heart attack? (Yes) 51.7

Do you think feeling weak, lightheaded, or faint is a symptom of a heart attack? (Yes) 67.4

Do you think chest pain or discomfort is a symptom of a heart attack? (Yes) 94.0

Do you think sudden trouble seeing in one or both eyes is a symptom of a heart attack? (No) 50.8

Do you think pain or discomfort in the arms or shoulders is a symptom of a heart attack? (Yes) 87.3

Do you think shortness of breath is a symptom of a heart attack? (Yes)

Stroke symptoms (correct answer)

Do you think sudden confusion or trouble speaking is a symptom of a stroke? (Yes) 94.2

Do you think sudden numbness or weakness of face, arm, or leg, especially on one side is a 96.5 symptom of a stroke? (Yes)

Do you think sudden trouble seeing in one or both eyes is a symptom of a stroke? (Yes) 81.7

Do you think sudden chest pain or discomfort is a symptom of a stroke? (No) 37.1

Do you think sudden trouble walking, dizziness, or loss of balance is a symptom of a stroke? (Yes) 91.6

Do you think severe headache with no known cause is a symptom of a stroke? (Yes) 58.3

Proper response to heart attack or stroke (correct answer)

If you thought someone was having a heart attack or a stroke, what is the first thing you would 86.8 do? (Call 911)

aWeighted $n=6,102,996$; unweighted $n=11,269$.

and stroke knowledge questions (range 0-8 points) were more likely to be aged $18-34$ rather than $\geq 55$ years $(\mathrm{OR}=1.36$, CI $1.35,1.37)$, be uninsured rather than insured $(\mathrm{OR}=1.32, \mathrm{CI} 1.31$,
1.33), have an annual household income $<\$ 35,000$ $(\mathrm{OR}=1.46, \mathrm{CI} 1.45,1.47)$, and have a primary healthcare provider $(\mathrm{OR}=1.22$, $\mathrm{CI}: 1.20 ; 1.23)$. Knowledge levels did not differ based on having

Table 3. Descriptive Statistics of Heart Attack and Stroke Knowledge

SCORes of African American Women $\geq 18$ Years of Age: 2003-2005 Behavioral Risk Factor Surveillance Survey Data

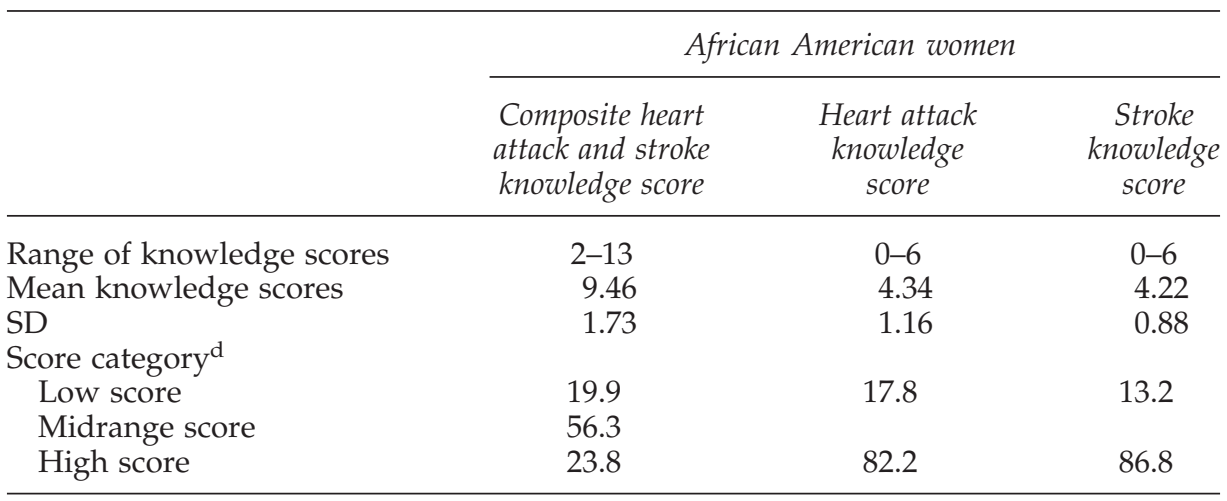

aWeighted $n=6,102,996$; unweighted $n=11,269$.

bThe composite heart attack and stroke knowledge score included the first reponder question. This was also included in the calculations for each domain's separate knowledge score.

cFor the heart attack and stroke knowledge scores calculated separately, a low and a high score were calculated using the following scale: low score $=0-4$ points or $\leq 50 \%$; high score $=5-7$ or $>50 \%$.

${ }^{\mathrm{d} C o r r e c t}$ answers received 1 point and were categorized according to the following scale for the composite scores: Low score $=0-8$ points or $\leq 60 \%$; midrange score $=9-10$ points or $69 \%-77 \%$; high score $=11-13$ points or $85 \%-100 \%$. 
Table 4. Bivariate Analysis of African American Women $\geq 18$ Years of Age by Independent Variables and Heart Attack and Stroke KnOWledge Score Level: 2003-2005 Behavioral Risk Factor Surveillance Survey Data

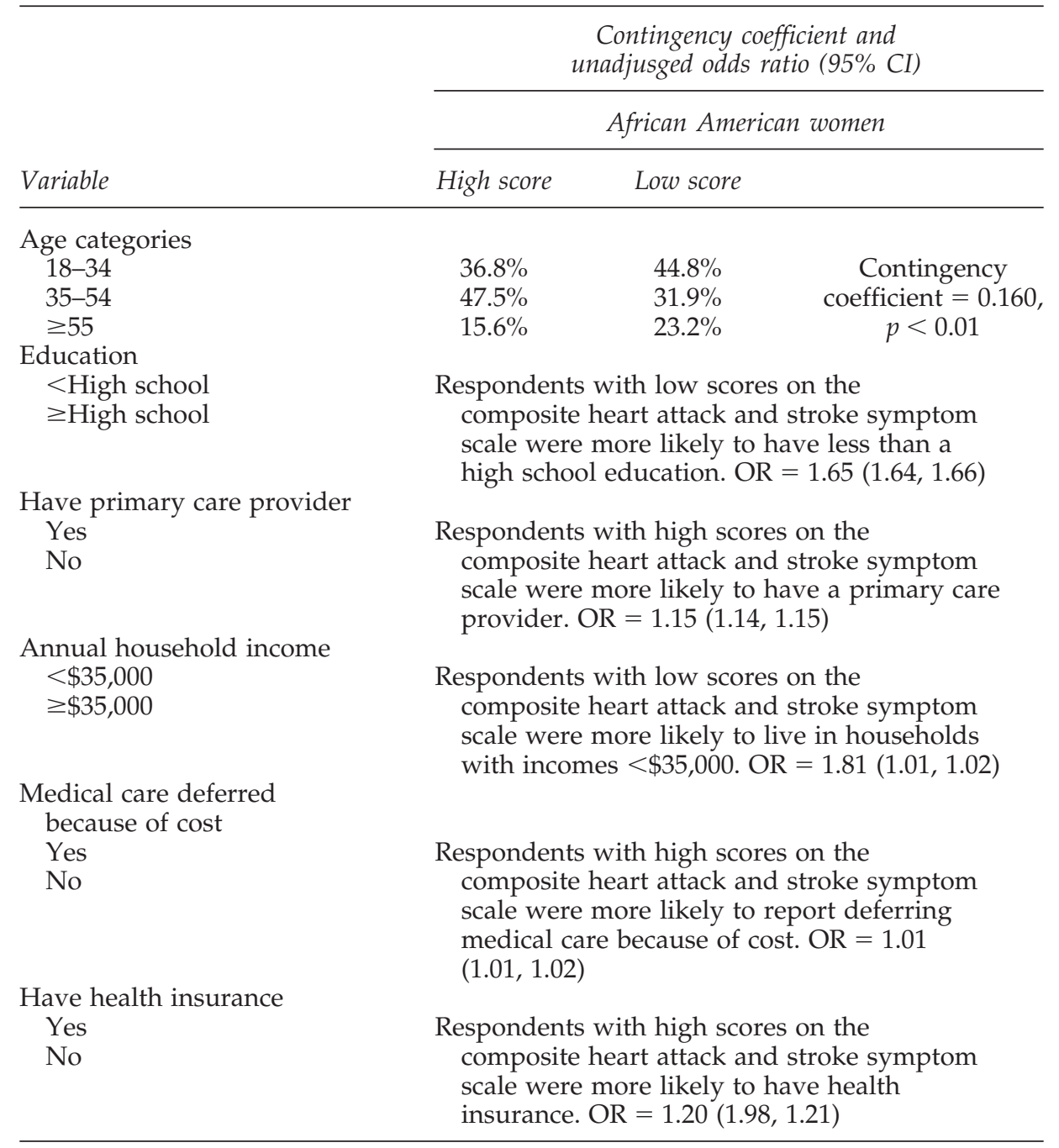

less than a high school education and not having deferred medical care because of cost.

\section{DISCUSSION}

The present study is an assessment of symptom awareness of heart attack and stroke in the adult African American female population using nationwide estimates by states. Our results indicated that African American women scored better on the stroke symptom knowledge questions than the heart attack symptom knowledge questions. These results differ from another recent study, the results of which indicated that women in general had better knowledge of heart attack symptoms that stroke symptoms. ${ }^{5}$ Methodologi- cal differences between the two studies might explain these contradictory results, as the data in our study used a forced choice answer format, whereas the other study generated data using an open-ended interview.

Our findings revealed that the majority of African American women scored in the moderate to high range for knowledge of heart attack and stroke symptoms. However, about 1 in 5 scored in the low range, suggesting that there is an opportunity for improving scores. For instance, even though $94 \%$ of respondents recognized that chest pain is a symptom of a heart attack, almost half did not think that pain or discomfort in the jaw, neck or back is a symptom of a heart attack. As women are more likely than men to have atypical symptoms of a heart attack, 
Table 5. Multivariate logistic Regression Results for Heart Attack and Stroke Low Knowledge Score for African American Women $\geq 18:$ 2003-2005 Behavioral Risk Factor Surveillance Survey Data

\begin{tabular}{lcr}
\hline Variable & Factors & $\begin{array}{c}\text { Adjusted odds ratio } \\
(95 \% \text { CI) }\end{array}$ \\
\hline Age categories (vs. $\geq 55)$ & $18-34$ & $1.36(1.35,1.37)$ \\
& $35-54$ & $0.87(0.86,0.88)$ \\
Education (vs. $\geq$ High school) & $<$ High school & $0.94(0.93,0.95)$ \\
Have health insurance (vs. Yes) & No & $1.32(1.31,1.33)$ \\
Annual household income (vs. $\geq \$ 35,000)$ & \$35,000 & $1.46(1.45,1.47)$ \\
Was medical care deferred because of & No & $1.08(1.07,1.09)$ \\
$\quad$ cost (vs. Yes) & Yes & $1.22(1.20,1.23)$ \\
\hline
\end{tabular}

this knowledge deficit is especially concerning. Consistent with previous studies, many women confused heart attack symptoms with stroke symptoms. ${ }^{18,21}$ Arguably, as either stroke or heart attack symptoms should trigger seeking care, mixing them up would likely have less impact than would failure to recognize atypical symptoms.

In addition, the study identified within-group distinctions and significant differences in knowledge of heart attack and stroke symptoms among adult African American women depending on socioeconomic variables and age. The association with socioeconomic status is consistent with the contention that socioeconomic status is a more powerful determinant of health disparities than race or ethnicity. Respondents earning low scores were $>40 \%$ more likely to have an annual household income $<\$ 35,000$, less likely to have health insurance, and more likely to be younger (ages 18-34). These results match results of previous studies indicating that younger age and lower socioeconomic status negatively influence knowledge of heart attack and stroke. ${ }^{22,23}$ They also identify at-risk subgroups so that measures might be specifically designed to enhance knowledge in high-risk adult African American females. Targeted interventions seem more likely to yield benefit than interventions aimed at the general adult African American female population.

Unexpectedly, individuals with lower scores were more likely to have primary healthcare providers. Findings from an earlier study indicated that primary care was significantly associated with lower stroke mortality. ${ }^{24}$ This earlier study, however did not examine knowledge of stroke symptomatology. Although it is unclear why women with a personal physician were less likely to be knowledgeable about symptoms, one possible explanation is that African American women may not feel as compelled to understand their health if they believe they have a physician to rely on. The surprising nature of this finding needs confirmation by future studies and also to determine if this relationship is unique to African American women. Nevertheless, it does highlight the primary care provider's role as an important source of information for patients and their responsibility for educating their patients about when to seek acute care.

Of course, knowledge about warning symptoms is only the first step required for successful early intervention. ${ }^{25}$ Following the patient recognition phase, subsequent steps include the prehospital phase and the hospital action phase, both of which the patient or bystander cannot control but are phases the patient must activate. ${ }^{25} \mathrm{Al}-$ though this study focused primarily on the patient recognition phase, the question concerning appropriate response to heart attack and stroke sheds light on patients' initiating action steps. Our study found that a higher proportion of African American women would call 911 if they thought someone was having a stroke or a heart attack than women in other studies. ${ }^{5}$ Previous studies also indicated that African American women underestimated their perceived cardiac risk, which can lead to treatment delays and may represent a barrier to taking action. ${ }^{16,25}$ In this study, almost 1 in 8 adult African American women answered incorrectly about calling 911 in response to someone having acute symptoms. Because transportation by ambulance for acute cardiovascular events is associated with earlier treatment and improved outcomes, ${ }^{26,27}$ educating this population about immediate contact with the emergency medical service upon recognition of warning symptoms of stroke and heart attack 
might accrue more benefit. In addition to knowledge, other barriers to activating emergency systems include such concerns as appearing foolish if the symptoms do not turn out to be serious. Future studies to identify these barriers should also prove useful in developing strategies for reducing treatment delays.

Several potential limitations to this study should be noted. First, the survey is based on telephone-derived data and may be skewed if those who did not participate were more likely to recognize symptoms. Even though the vast majority of Americans live in households with phones (landlines or cell), it is nevertheless still the case that those without are the poorest households, with the least educated occupants who are ethnic minorities (Hispanic or African American) ${ }^{28}$ As lower socioeconomic status correlates with lower symptom awareness, our findings could overestimate knowledge or the gap between lower socioeconomic status African American women. The bias introduced into the study by the issues associated with phone coverage, however, cannot be changed. A second limitation is that the survey consists of closed-ended questions, and this may cause an overestimation of knowledge. A different format to the survey may have yielded very different results. Third and finally, it is possible that the nonresponders to the BRFSS might have scored differently on the questions, skewing the results. ${ }^{7}$ On the positive side, a strength of the study is the large number of individuals surveyed, yielding a nationally representative sample.

Symptom recognition for stroke and heart attack is a critical first step for early treatment. Overall knowledge of symptoms among adult African American women is good; however, socioeconomic variables identify subgroups in which the knowledge level is far from optimal. The next important step is to tailor interventional programs specific to these high-risk subgroups to improve symptom recognition. Future educational initiatives and programs should be targeted to patients and their physicians to improve heart attack and stroke symptom awareness, especially among African American women, who are at higher risk for poor clinical outcomes.

\section{DISCLOSURE STATEMENT}

No competing financial interests exist.

\section{REFERENCES}

1. Thom T, Haase N, Rosamond W, et al. Heart disease and stroke statistics-2006 update: A report from the American Heart Association statistics committee and stroke statistics subcommittee. Circulation 2006;113: e85.

2. Miniño AM, Heron MP, Murphy SL, Kochankek, KD. Deaths: Final data for 2004. National vital statistics reports. Hyattsville, MD: National Center for Health Statistics, 2007;55(19).

3. Panicioli AM, Broderick J, Kothari R, et al. Public perception of stroke warning signs and knowledge of potential risk factors. JAMA 1998;279:1288.

4. Greenlund KJ, Neff LJ, Zheng Z, et al. Low public recognition of major stroke symptoms. Am J Prev Med 2003;25:315.

5. Christian AH, Rosamond W, White AR, Mosca L. Nine-year trends and racial and ethnic disparities in women's awareness of heart disease and stroke: An American Heart Association national study. I Womens Health 2007;16:68.

6. De Luca, G, Suryapranata, H, Ottervanger, JP, Antman, EM. Time delay to treatment and mortality in primary angioplasty for acute myocardial infarction: Every minute of delay counts. Circulation 2004;109:1223.

7. Greenlund KJ, Denny CH, Mokdad AH, Watkins N, Croft JB, Mensah GA. Using Behavioral Risk Factor Surveillance data for heart disease and stroke prevention programs. Am J Prev Med 2005;29:81.

8. Greenlund KJ, Keenan NL, Giles WH, et al. Public recognition of major signs and symptoms of heart attack: Seventeen states and the U.S. Virgin Islands, 2001. Am Heart J 2004;147:1010.

9. Berger PB, Ellis SG, Holmes DR, et al. Relationship between delay in performing direct coronary angioplasty and early clinical outcome in patients with acute myocardial infarction. Circulation 1999;100:14.

10. Lisboa RC, Jovanovic BD, Alberts MJ. Analysis of the safety and efficacy of intra-arterial thrombolytic therapy in ischemic stroke. Stroke 2002;33:2866.

11. Davalos A. Thrombolysis in acute ischemic stroke: Successes, failures, and new hopes. Cerebrovasc Dis 2005;20(Suppl 2):135.

12. Lewandowski CA, Frankel M, Tomsick TA, et al. Combined intravenous and intra-arterial r-TPA versus intra-arterial therapy of acute ischemic stroke. Stroke 1999;30:2598.

13. Lacy CR, Suh DC, Bueno M, Kostis JB. Delay in presentation and evaluation for acute stroke: Stroke Time Registry for Outcomes Knowledge and Epidemiology (S.T.R.O.K.E.). Stroke 2001;32:63.

14. Murphy SA, Chen C, Canoon CP, Antman EM, Gibson CM. Impact of gender on angiographic and clinical outcomes after fibrinolytic therapy in acute myocardial infarction. Am J Cardiol 2002;90:766.

15. Rosenfeld AG. Treatment-seeking delay among women with acute myocardial infarction: Decision trajectories and their predictors. Nurs Res 2004;53:225. 
16. DeSalvo KB, Gregg J, Kleinpeter M, Pederson BR, Stepter A, Peabody J. Cardiac risk underestimation in urban, black women. J Gen Intern Med 2005;20:1127.

17. Zerwic J, Ryan C, DeVon H, Drell M. Treatment seeking for acute myocardial infarction symptoms: Differences in delay across sex and race. Nurs Res 2003;52:159.

18. Aslanian-Engoren C. Black, Hispanic, and white women's knowledge of the symptoms of acute myocardial infarction. JOGNN. 2005;34:505.

19. Mandelzweig L, Goldbourt U, Boyko V, Tanne D. Perceptual, social, and behavioral factors associated with delays in seeking medical care in patients with symptoms of acute stroke. Stroke 2006;37:1248.

20. Centers for Disease Control and Prevention. Behavioral Risk Factor Surveillance System users' guide. Atlanta, GA: U.S. Department of Health and Human Services, Centers for Disease Control and Prevention, 1998.

21. Reeves MJ, Hogan JG, Rafferty AP. Knowledge of stroke risk factors and warning signs among Michigan adults. Neurology 2002;59:1547.

22. Goff D, Seller D, McGovern P, et al. Knowledge of heart attack symptoms in a population survey in the United States. Arch Intern Med 1998;158:2329.

23. Mosca L, Jones WK, King KB, Ouyang P, Redberg RF, Hill MN. Awareness, perception, and knowledge of heart disease risk and prevention among women in the United States. Arch Fam Med 2000;9:506.

24. Leiyu S, Macinko J, Starfield B, Xu J, Politzer R. Primary care, income inequality, and stroke mortality in the United States: A longitudinal analysis, 1985-1995. Stroke 2003;34:1958.

25. Harralson TL. Factors influencing delay in seeking treatment for acute ischemic symptoms among lower income, urban women. Heart Lung 2007;36:96.

26. Schwamm LH, Pancioli A, Acker JE, et al.; Task Force Members. Recommendations for the establishment of stroke systems of care: Recommendations from the American Stroke Association's task force on the development of stroke systems. Stroke 2005;36:690.

27. U.S. Department of Health and Human Services. Healthy people 2010, 2nd ed. With understanding and improving health and objectives for improving health (2 vols.). Washington, DC: U.S. Department of Health and Human Services, 2000.

28. Blumberg SJ, Luke JV, Cynamon ML. Telephone coverage and health survey estimates: Evaluating the need for concern about wireless substitution. Am J Public Health 2006;96:926.

Address reprint requests to: May Nawal Lutfiyya, Ph.D. Department of Family and Community Medicine University of Illinois-Chicago College of Medicine at Rockford Rockford, IL 61107

E-mail: lutfiyya@uic.edu 
This article has been cited by:

1. May Nawal Lutfiyya, Ricardo Bardales, Robert Bales, Carlos Aguero, Shelly Brady, Adriana Tobar, Cynthia McGrath, Julia Zaiser, Martin S. Lipsky. 2009. Awareness of Heart Attack and Stroke Symptoms Among Hispanic Male Adults Living in the United States. Journal of Immigrant and Minority Health . [CrossRef] 